\title{
Impact of Ramadan on Physical Activity and Sleeping Patterns in Individuals with Type 2 Diabetes: The First Study Using Fitbit Device
}

\author{
Abdullah S. Alghamdi (D) - Khalid A. Alghamdi • Richard O. Jenkins • \\ Mohammed N. Alghamdi · Parvez I. Haris
}

Received: March 26, 2020 / Published online: May 4, 2020

(C) The Author(s) 2020

\begin{abstract}
Introduction: The impact of Ramadan fasting, a type of intermittent fasting, on the management of diabetes has not been well investigated. Physical activity, sleep duration, and time of sleep are susceptible to alterations during Ramadan due to the changes in the times and numbers of meals. This study compared physical activity and sleep patterns of individuals with type 2 diabetes mellitus (T2DM) during and after Ramadan using the international physical activity questionnaire (IPAQ) and a Fitbit Flex 2 accelerometer.
\end{abstract}

Digital Features To view enhanced digital features for this article go to https://doi.org/10.6084/m9.figshare. 12155568.

Electronic Supplementary Material The online version of this article (https://doi.org/10.1007/s13300020-00825-x) contains supplementary material, which is available to authorized users.

A. S. Alghamdi $(\bowtie) \cdot$ R. O. Jenkins · P. I. Haris Faculty of Health and Life Sciences, De Montfort University, Leicester, UK

e-mail: ph-abdallah@hotmail.com;

P15017435@my365.dmu.ac.uk

\section{A. S. Alghamdi}

General Directorate of Medical Services of the Interior Ministry, Riyadh, Saudi Arabia

K. A. Alghamdi · M. N. Alghamdi

Al Iman General Hospital, Riyadh, Saudi Arabia
Methods: Saudi individuals $(n=36)$ with T2DM completed a self-reported questionnaire and wore a Fitbit device for seven consecutive days during and after Ramadan. Fitbit generated weekly step counts, activity intensities, sedentary time, and sleep durations and times. IPAQ was used to estimate the physical activity and sitting time of participants in each period. Sleep patterns were assessed in each period by a selfreported questionnaire.

Results: Both Fitbit and IPAQ indicated a high prevalence of low physical activity among the participants with non-significant variances between the during and after Ramadan periods. Also, a significant short daily total sleeping hours and daily night-time sleeping hours was seen during the Ramadan period. The duration of night-time sleep was observed to be low in each period.

Conclusions: This is the first study to use a Fitbit device to monitor individuals with T2DM who chose to fast during Ramadan. The study shows a high prevalence of low physical activity among Saudi individuals with T2DM in each period, and short sleep durations in the during Ramadan period compared to after Ramadan period. A high prevalence of short night-time sleep duration and excessive daytime sleeping was observed in both periods and significantly in the during Ramadan period. A larger study is needed in the future covering before, during, and after Ramadan to evaluate the impact of lifestyle changes related to Ramadan fasting on type 2 diabetes. 
Keywords: Diabetes; Fitbit; IPAQ; Physical activity; Ramadan; Sleep; T2DM

\section{Key Summary Points}

Why carry out this study?

Saudi Arabia has one of the highest prevalences of diabetes in the world with over $30 \%$ of its population affected by type 2 diabetes. This has negative impacts on the lives of individuals and is a great burden on the healthcare system and the economy of the country.

Physical activity and sleeping patterns are important factors for managing diabetes. These are susceptible to alterations during the month of Ramadan when most adult Saudis perform a type of intermittent fasting, from dawn till dusk for 29-30 days. How Ramadan fasting alters sleeping and physical activity of Saudis with type 2 diabetes has not been well investigated.

\section{What was learned from the study?}

High prevalence of physical inactivity among study participants is a cause for concern and further research is needed in this area with a larger population.

Short sleeping duration during Ramadan and prevalence of short night-time sleep after Ramadan may have adverse effects on the health of individuals with type 2 diabetes which needs to be further investigated.

\section{INTRODUCTION}

Diabetes mellitus is a common chronic disease, which has been estimated to affect $31.6 \%$ of the Saudi population [1]. Different factors, such as environmental, social, and lifestyle factors, have been linked with the treatment and incidence of type 2 diabetes mellitus (T2DM). For instance, several studies have demonstrated that increased physical activity and enhanced sleep quality are essential factors for better outcomes in the control of T2DM [2-6]. Increased physical activity has been reported to be correlated with reduced HbA1c and glycaemic levels, and decreased cardiovascular complications among individuals with T2DM [7-9]. The benefits of physical activity on T2DM have been shown through the significant improvements in the sensitivity of insulin [10]. However, physical inactivity can increase the susceptibility of developing of T2DM or worsen the diabetic condition in individuals who are already suffering from this condition $[3,6,11]$. Unfortunately, physical inactivity has been reported to be common among the Saudi population, where the General Authority for Statistics in Saudi Arabia recently reported that only $17.4 \%$ of Saudis are performing physical activity for $150 \mathrm{~min}$ or more per week [12].

For the sleep quality, both long and short sleep durations have been previously reported to be associated with increased HbA1c levels $[13,14]$. Sleep during the night-time is important for the metabolic process, which has been shown to facilitate blood glucose regulation that is essential for diabetes outcomes [15]. Short duration of sleeping during the nighttime has been observed among a portion of the Saudi population $[16,17]$. In addition, numerous studies have shown that the disturbance of the circadian rhythm can cause T2DM or make it worsen through affecting glucose homeostasis, insulin secretion, and insulin sensitivity [18-20].

The month of Ramadan is the ninth month in the lunar year, in which healthy adult Muslims abstain from any type of food, drink, or medicine administered through the mouth, smoking, and sexual interaction from dawn to dusk each day for 29 or 30 days [21]. As a result of some activities during the month of Ramadan and changes in the numbers and times of meals, Ramadan has been reported to be associated with alterations in sleeping durations and times, and physical activities [22-27]. These changes may affect public health, and 
particularly individuals with diabetes. At worst, these may minimise the achievement of optimal positive impact of intermittent fasting during Ramadan, which has been previously reported to be beneficial for T2DM [28-31]. Therefore, the investigation of the physical activity and sleeping patterns of Muslim individuals with T2DM during Ramadan, using a precise measurement tool, is needed to help understand the impact of Ramadan fasting. However, the precise measurement of the complex set of behaviours such as physical activity and sleeping patterns is not easy as selfreported questionnaires, which are the most common measurement tools, are susceptible to recall bias and misreporting [32-34]. Wearable devices such as Fitbit have been previously reported to provide reliable assessment of physical activity and sleep $[35,36]$. Hence, this study aimed to investigate and evaluate the physical activity and sleeping patterns of Saudi individuals with type 2 diabetes during and after Ramadan periods using the Fitbit device and a questionnaire. The outcomes of physical activity and sleeping patterns during the Ramadan period were compared to those in the after Ramadan period. In addition, the outcomes of each method were correlated with those of the other method, and the significance and direction of these correlations were evaluated.

\section{METHODOLOGY}

\section{Study Settings and Design}

This was an exploratory pilot study conducted over two periods (during and after Ramadan). In this study, 36 adult Muslims with T2DM, who had an intention to fast during Ramadan, were recruited during their routine visits to the Endocrine and Diabetic Centre of Al Iman General Hospital, Riyadh, Saudi Arabia. The during Ramadan visit occurred in the middle of the month of Ramadan [29 May-14 June, 2018], and the after Ramadan visit was arranged 2 weeks after the end of the month of Ramadan [29 Jun-13 July, 2018]. Informed consent was obtained from each participant prior to commencement of the study. Baseline demographic data was collected in the during Ramadan visit. Self-reported baseline demographic data were collected from each participant. In each period, two different techniques, namely self-reported questionnaire and Fitbit Flex 2 accelerometer (Fitbit Inc., San Francisco, CA), were used to assess the physical activity and sleeping patterns of the participants. Along with physical measurements for each participant, blood samples after $12 \mathrm{~h}$ of fasting were obtained for laboratory analysis.

\section{Data Collection and Measurements}

\section{Demographic Data}

Demographic data were collected by self-reported questionnaire in the first visit. Demographic data included age, gender, marital status, employment status, and duration of T2DM.

\section{Physical and Laboratory Measurements}

In each period, height (to the nearest $0.5 \mathrm{~cm}$ ), weight (to the nearest $0.1 \mathrm{~kg}$ ), and waist size (to the nearest $0.5 \mathrm{~cm}$ ) were measured for each participant. The waist size was measured with a tape placed in the middle between the lower margin of the ribs and the superior border of the iliac crest. Body mass index (BMI) was calculated for each participant, using the ratio of weight (in kilograms) to the height (in metres) squared. Blood samples were determined in the main laboratory of $\mathrm{Al}$ Iman General Hospital for measuring HbA1c. All blood samples were analysed using a biochemical analyser (Dimension $\mathrm{RxL} \mathrm{Max}^{\circledR}$, Siemens Medical Solution Diagnostics, Tarrytown, NY) in accordance with the instructions provided by the manufacturer.

\section{Physical Activity}

Physical activity data for weekly step counts, different intensity of activity, walking, and sedentary and sitting times were measured using a Fitbit Flex 2 accelerometer and a selfreported questionnaire. The self-reported questionnaire was supported by using the Arabic version of the short form of the international physical activity questionnaire (IPAQ). 


\section{Fitbit Device}

The Fitbit Flex 2 accelerometer is a wearable and waterproof device that tracks physical activity and sleep, and can store data for 7 days without syncing [37]. Each device was linked to an account created on the Fitbit website (https:// www.fitbit.com), and the saved data were exported from the Fitbit online dashboard. The active minutes of $10 \mathrm{~min}$ duration or more are calculated by Fitbit as metabolic equivalents (METs) and presented in minutes per week. Each participant was asked to wear the Fitbit Flex 2 device for seven consecutive days before each visit (during and after Ramadan). Participants were informed to wear the device all the time during each period, and to keep their regular activities. In addition, no instructions or specific goals were given to the participants regarding their physical activities or sleep. Raw Fitbit data of 7 days were collected at the end of each period. The raw data included step counts, low activity, moderate activity, vigorous activity, and sedentary time. After collecting all the required data, all accounts were deleted from the Fitbit website and all devices were reset to avoid further data collection.

\section{IPAQ}

IPAQ was created in 1997 as an observational measurement to assess physical activity, which was validated and reported with high reliability $[38,39]$. The short form of IPAQ is composed of seven questions that are used to estimate the time spent per week performing different intensities of physical activity. This physical activity can be categorized according to its intensity, whether vigorous, moderate, or low. These categories are estimated using a metabolic equivalent (MET) value and presented as MET$\mathrm{min} /$ week. The IPAQ estimates the types of the intensity of physical activity, walking, and sitting time of participants during a usual week.

\section{Sleeping Patterns}

A self-reported questionnaire with closed-ended questions was used in each period to assess the sleeping patterns of participants through asking about the total number of sleeping hours per day, the number of night-time sleeping hours, and the total number of hours spent sleeping daily. In addition, the Fitbit Flex 2 accelerometer was used to record sleep durations and times for each participant in each period. The sleep data from the Fitbit device was obtained by recording the duration of sleep between sunset and sunrise, according to the location of the study. This was considered as night-time sleep for each participant. Consequently, the daily averages of total sleeping hours and night-time sleeping hours were calculated for each participant. In addition, the average daily total sleeping hours and average daily night-time sleeping hours were organised into different categories.

\section{Statistical Analysis}

Statistical analysis was performed using SPSS software version 25 (IBM Corporation, Armonk, NY, USA). Descriptive statistics analysis was applied for outcomes where mean \pm standard deviation (SD) was used for normally distributed continuous variables, median and interquartile range (IQR) was used for nonnormally distributed continuous variables, and counts and percentages were used for categorical variables. The normality of each continuous variable was assessed. For the normally distributed variable, paired $t$ test was applied to compare two variables between the two periods, and one-way ANOVA was used to detect variances between groups. For the non-normally distributed variables, non-parametric tests such as Wilcoxon and Spearman correlation were used in the analysis. The effect size $(r)$ was also calculated to measure the magnitude of the differences between periods. This effect size (ES) was calculated by the following formula: $r=Z$ / $\sqrt{ } n$ (where $Z=Z$ score produced by Wilcoxon signed rank test and $n=$ total sample size). The obtained ES was reported according to Cohen's [40] guidelines $(0.1,0.3$, and 0.5 indicating small, moderate, and large effects, respectively). A chi-square test was used to compare the variance in the proportion between categorical variables. If any cell of the chi-square test was less than 5, the Fisher's exact test was conducted. The statistical significance level for all two-tailed tests was set at $P<0.05$. 
Ethical approvals were obtained from the Faculty of Health and Life Sciences Research Ethics Committee at De Montfort University and the Ministry of Health in Saudi Arabia. All procedures followed were in accordance with the Helsinki Declaration of 1964 and its later amendments. Informed consent was obtained from each participant prior to commencement of the study to take part in this study and for any publication with non-identifiable quotes.

\section{RESULTS}

The baseline sociodemographic characteristics of the 36 participants are presented in Table 1 , of whom $55.6 \%(n=20)$ were men and $44.4 \%$ $(n=16)$ were women. The mean age \pm SD of the participants was $49.50 \pm 11.91$ years. Fifty percent of participants were aged 41-60 years and $80.6 \%$ of the participants were married. There was a significant variance between genders regarding their employment status $\left(x^{2}=15.59 ; P<0.001\right)$, as most of the employed participants were men. The mean duration of having T2DM was $10.92 \pm 8.88$ years for the total study sample.

Table 2 demonstrates the comparisons of the physical measurements and laboratory results of the participants between the during and after Ramadan periods. No significant variances were found between the during and after Ramadan periods in the means of weight, BMI, waist size, and HbA1c levels. Only one participant had normal BMI, while more than half of the participants were obese. Interestingly, HbA1c levels during Ramadan were lower than in the after Ramadan period but the difference was neither statistically significant nor clinically important.

The comparisons of the obtained data regarding the physical activities from IPAQ and Fitbit Flex 2 in the two different periods are shown in Table 3 . The Wilcoxon signed ranks tests revealed no statistically significant variances between the during and after Ramadan periods in all types of participants' activities and sitting or sedentary times with very small effect sizes. However, the difference between periods in the total activity (vigorous + moderate + walking) measured by IPAQ was statistically significant $(P=0.029)$ but with small ES $(r=0.257)$. Although the levels of the majority of participants were low according to IPAQ in both periods, the during Ramadan period showed slightly higher physical activity but was not significant compared to the after Ramadan period $(P=0.560)$. Low level of physical activity was observed in at least $69.4 \%$ of participants in each period.

Table 4 presents the Spearman's rank correlations between the physical activity outcomes of the Fitbit and the IPAQ in each period. The analysis revealed that there were strong positive significant correlations between vigorous activities and moderate activities measured by Fitbit Flex 2 and IPAQ during Ramadan that became moderate correlations in the after Ramadan period. The step counts by Fitbit strongly correlated with the walking measured by IPAQ in each period (during and after Ramadan). For the sedentary and sitting times, there were moderate correlations between the measurements of Fitbit and IPAQ in each period (during and after Ramadan). In addition, the sedentary and sitting times measured by the Fitbit and the IPAQ negatively correlated with all other activity types in each period. The daily average of sitting and sedentary times, measured by both instruments, were at least $10 \mathrm{~h}$ in each period. In addition, the sitting time obtained by the IPAQ was under-reported by participants compared to sedentary time collected by Fitbit Flex 2 in each period $(P<0.05)$.

The outcomes regarding sleeping patterns of participants, reported by questionnaire and recorded by Fitbit Flex 2, are shown in Table 5 . The statistical analysis demonstrates significant differences between the during Ramadan and after Ramadan periods in the sleeping patterns measured by both methods, with reduced sleeping hours during the Ramadan period. According to the Fitbit, the median daily total sleeping hours significantly increased in the after Ramadan period compared to the during Ramadan period $(7.02 \mathrm{~h}$ vs $6.03 \mathrm{~h} ; P<0.001)$, with moderate ES $(r=0.457)$. The average daily total sleeping hours of less than $6 \mathrm{~h}$, recorded by Fitbit, was observed in $50 \%$ of participants during Ramadan and in 13.9\% after Ramadan. Interestingly, half or more of participants did 
Table 1 Baseline sociodemographic characteristics of participants $(n=36)$

\begin{tabular}{|c|c|c|c|c|}
\hline \multirow[t]{2}{*}{ Outcomes } & \multicolumn{3}{|c|}{ Mean \pm SD or $n(\%)$} & \multirow[t]{2}{*}{$P$ value } \\
\hline & Total $(n=36)$ & Male $(n=20)$ & Female $(n=16)$ & \\
\hline Age (years) & $49.50 \pm 11.91$ & $49.25 \pm 11.48$ & $49.81 \pm 12.80$ & 0.890 \\
\hline \multicolumn{5}{|l|}{ Age group (years) } \\
\hline$\leq 40$ & $9(25 \%)$ & $4(20 \%)$ & $5(31.3 \%)$ & \multirow[t]{3}{*}{$0.457^{*}$} \\
\hline $41-60$ & $18(50 \%)$ & $12(60 \%)$ & $6(37.5 \%)$ & \\
\hline$>60$ & $9(25 \%)$ & $4(20 \%)$ & $5(31.3 \%)$ & \\
\hline Diabetes duration (years) & $10.92 \pm 8.88$ & $10.35 \pm 8.57$ & $11.63 \pm 9.50$ & 0.675 \\
\hline \multicolumn{5}{|l|}{ Marital status } \\
\hline Married & $29(80.6 \%)$ & $18(90 \%)$ & $11(68.8 \%)$ & \multirow[t]{3}{*}{$0.060^{\prime}$} \\
\hline Single & $3(8.3 \%)$ & $2(10 \%)$ & $1(6.3 \%)$ & \\
\hline Divorced/widowed & $4(11.1 \%)$ & $0(0.0)$ & $4(25 \%)$ & \\
\hline \multicolumn{5}{|l|}{ Employment status } \\
\hline Employed & $13(36.1 \%)$ & $11(55 \%)$ & $2(12.5 \%)$ & \multirow[t]{3}{*}{$<0.001^{\prime}$} \\
\hline Unemployed & $16(44.4 \%)$ & $3(15 \%)$ & $13(81.3 \%)$ & \\
\hline Retired & $7(19.4 \%)$ & $6(30 \%)$ & $1(6.3 \%)$ & \\
\hline
\end{tabular}

$P$ values of the unpaired $t$ tests for continuous variables or of the chi-square test for categorical variables

${ }^{*}$ A Fisher's exact test was applied if any cell of chi-square test was $<5$

Table 2 Comparisons of physical measurements and laboratory results of the participants between the during and after Ramadan periods $(n=36)$

\begin{tabular}{lllll}
\hline & \multicolumn{2}{l}{ Mean \pm SD or $\boldsymbol{n}(\%)$} & 95\% CI & P value \\
\cline { 2 - 4 } & During Ramadan & After Ramadan & & \\
\hline Weight $(\mathrm{kg})$ & $85.76 \pm 17.38$ & $85.73 \pm 16.01$ & -2.13 to 2.07 & 0.976 \\
BMI & $32.89 \pm 5.47$ & $32.94 \pm 5.12$ & -0.71 to 0.81 & 0.892 \\
BMI categories & & & \\
Normal & $1(2.78 \%)$ & $1(2.78 \%)$ & $1.000^{*}$ \\
Overweight & $13(36.11 \%)$ & $14(38.89 \%)$ & \\
Obese & $22(61.11 \%)$ & $21(58.33 \%)$ & & \\
Waist size $(\mathrm{cm})$ & $107.00 \pm 11.78$ & $106.10 \pm 11.15$ & -2.60 to 0.82 & 0.296 \\
HbAlc $(\%)$ & $7.81 \pm 1.40$ & $7.92 \pm 1.38$ & -0.02 to 0.23 & 0.108 \\
\hline
\end{tabular}

$P$ values of the paired $t$ tests for continuous variables and of the chi-square test for categorical variables $S D$ standard deviation, $C I$ confidence intervals, $B M I$ body mass index, $H b A l c$ glycated haemoglobin

${ }^{*}$ A Fisher's exact test was applied if any cell of chi-square test was $<5$ 
Table 3 Comparisons between the physical activity outcomes measured by IPAQ and Fitbit Flex 2 for participants in the two different periods (during and after Ramadan, $n=36$ )

\begin{tabular}{lcccc}
\hline Outcomes & \multicolumn{2}{l}{ Median (IQR) or $\boldsymbol{n}(\%)$} & \multirow{2}{*}{$\boldsymbol{P}$ value } & SE $(\boldsymbol{r})$ \\
\cline { 2 - 3 } & During Ramadan & After Ramadan & & \\
\hline IPAQ (MET-min/week) & $0(0-240)$ & $0(0-240)$ & 0.095 & 0.196 \\
Vigorous activity & $120(40-180)$ & $120(40-120)$ & 0.160 & 0.166 \\
Moderate activity & $396(297-495)$ & $396(231-495)$ & 0.882 & 0.018 \\
Walking activity & $676(414-930)$ & $516(351-855)$ & 0.029 & 0.257 \\
Total (vigorous + moderate + walking) & $74(70-88)$ & $73.5(63-87.5)$ & 0.750 & 0.037 \\
Sitting time (h/week) & & & \\
IPAQ level & $25(69.4 \%)$ & $28(77.8 \%)$ & 0.560 & \\
Low & $7(19.4 \%)$ & $5(13.9 \%)$ & & \\
Moderate & $4(11.1 \%)$ & $3(8.3 \%)$ & & \\
High & & & & \\
Fitbit Flex 2 (min/week) & $148(0-204)$ & $66(0-141)$ & 0.092 & 0.199 \\
Vigorous activity & $70(30-209)$ & $116(38-202)$ & 0.829 & 0.025 \\
Moderate activity & $1403(1104-1819)$ & $1559(1139-1726)$ & 0.203 & 0.150 \\
Low activity & $32,170(28,953-55,632)$ & $36,681(28,449-50,964)$ & 0.326 & 0.116 \\
Steps per week & $34,084(30,157-57,787)$ & $38,480(29,612-52,979)$ & 0.451 & 0.089 \\
Total (vigorous + moderate + low + steps) & $97(92.3-118)$ & $99.5(88-102)$ & 0.102 & 0.193 \\
Sedentary (h/week) & & &
\end{tabular}

$P$ values for nonparametric paired Wilcoxon signed ranks tests

$I P A Q$ international physical activity questionnaire, MET metabolic equivalents, $n$ number, $I Q R$ interquartile range, $E S(r)$ effect size $r$

not report any instances of all sleeping being at night-time during the Ramadan period by both measurement methods. In the after Ramadan period, sleeping during night-time significantly increased compared to during Ramadan, with moderate ES $(r=0.439)$. However, it was still low with sleeping of less than $3 \mathrm{~h}$ at night-time being reported by $33.33 \%$ of participants in the questionnaire and $77.8 \%$ in the Fitbit. The statistical analysis did not detect significant correlations between the average daily total sleeping hours, recorded by Fitbit and reported in the questionnaire, during and after Ramadan periods $\left(r_{\mathrm{s}}=0.321, n=36, P=0.056\right.$ and $r_{\mathrm{s}}=$ $0.242, \quad n=36, \quad P=0.155, \quad$ respectively).
Additionally, all these non-significant correlations were week correlations. Likewise, the average daily night-time sleeping hours, recorded by the Fitbit, did not significantly correlate to that reported by questionnaire in the during and after Ramadan periods $\left(r_{\mathrm{s}}=0.212, n=36\right.$, $P=0.214$ and $r_{\mathrm{s}}=0.235, \quad n=36, P=0.169$, respectively). Furthermore, all these non-significant correlations were week correlations. From the former analysis and correlations, there was over-reporting of outcomes by participants in the questionnaire compared to Fitbit outcomes regarding their daily total sleeping hours and daily night-time sleeping hours in both periods (during and after Ramadan). 
Table 4 Spearman's rank correlations coefficients $\left(r_{\mathrm{s}}\right)$ between the physical activity outcomes measured by Fitbit Flex 2 and IPAQ in each period (during and after Ramadan, $n=36$ )

\begin{tabular}{|c|c|c|c|c|c|c|c|c|}
\hline & \multicolumn{4}{|c|}{ During Ramadan } & \multicolumn{4}{|c|}{ After Ramadan } \\
\hline & \multicolumn{4}{|l|}{ IPAQ } & \multicolumn{4}{|l|}{ IPAQ } \\
\hline & $\begin{array}{l}\text { Vigorous } \\
\text { activity }\end{array}$ & $\begin{array}{l}\text { Moderate } \\
\text { activity }\end{array}$ & Walking & $\begin{array}{l}\text { Sitting (h/ } \\
\text { week) }\end{array}$ & $\begin{array}{l}\text { Vigorous } \\
\text { activity }\end{array}$ & $\begin{array}{l}\text { Moderate } \\
\text { activity }\end{array}$ & Walking & $\begin{array}{l}\text { Sitting }(\mathrm{h} / \\
\text { week) }\end{array}$ \\
\hline \multicolumn{9}{|l|}{ Fitbit Flex 2} \\
\hline $\begin{array}{l}\text { Vigorous } \\
\text { activity }\end{array}$ & $0.643^{*}$ & $0.507^{*}$ & 0.200 & -0.225 & $0.341^{*}$ & 0.250 & $0.711^{* *}$ & -0.233 \\
\hline $\begin{array}{l}\text { Moderate } \\
\text { activity }\end{array}$ & 0.131 & $0.792^{*}$ & $0.502^{*}$ & $-0.461^{*}$ & 0.242 & $0.452^{*}$ & $0.643^{*}$ & $-0.353^{*}$ \\
\hline $\begin{array}{l}\text { Steps (per } \\
\text { week) }\end{array}$ & 0.249 & $0.637^{*}$ & $0.743^{*}$ & $-0.474^{*}$ & $0.343^{*}$ & $0.511^{*}$ & $0.806^{*}$ & $-0.396^{*}$ \\
\hline $\begin{array}{r}\text { Sedentary } \\
\text { (h/week) }\end{array}$ & -0.189 & $-0.397^{*}$ & $-0.541^{*}$ & $0.344^{*}$ & -0.207 & $-0.401^{*}$ & $-0.436^{*}$ & $0.460^{*}$ \\
\hline
\end{tabular}

$I P A Q$ international physical activity questionnaire, $M E T$ metabolic equivalents

${ }^{*}$ Correlation is significant with $P<0.05$

Independent $t$ test and one-way ANOVA tests did not detect any statistically significant variances between genders, age groups, marital status, and employment status in the total activity measured by both methods for each period $(P>0.05)$. Similarly, no statistically significant differences were detected in mean HbA1c levels between different IPAQ levels, i.e. low, moderate, and high $(P>0.05)$. Furthermore, mean HbA1c levels did not significantly vary between different sleeping patterns reported by both methods in each period. Interestingly, Spearman's rank indicated that HbA1c levels negatively but not-significantly correlated with the total activity by IPAQ during and after Ramadan, and total activity by Fitbit during and after Ramadan periods $(P=0.394, P=0.766$, $P=0.695$, and $P=0.680$; respectively).

\section{DISCUSSION}

Ramadan fasting is a type of intermittent fasting that is performed for 1 month by Muslims around the world. Although intermittent fasting has been reported to have diverse health benefits [41-45], research on Ramadan fasting is limited. Our study reveals a high prevalence of physical inactivity among participants in the during and after Ramadan periods obtained by a wearable device (Fitbit) and a self-reported questionnaire. In addition, a high prevalence of sedentary and sitting times was recorded by both methods in each period. These findings are of concern since it has been previously reported that these behaviours negatively impact on the management and treatment of diabetes [3, 6], or are linked to the development of T2DM [11, 46]. Moreover, a positive correlation was found between increased sedentary time and some risk factors of developing T2DM such as increased waist size and BMI [47]. In contrast, increased daily steps, which are considered a type of physical activity, was reported to be associated with reduced HbA1c levels [9]. Physical activity can increase glucose uptake by muscles and organs of the body in order to produce energy without the need for insulin [48].

Our findings of a high prevalence of physical inactivity and increased sedentary time are consistent with previous studies that have reported low physical activity among Saudis. 
Table 5 Comparisons between the sleeping outcomes collected by questionnaire and Fitbit Flex 2 for participants in the two different periods (during and after Ramadan, $n=36$ )

\begin{tabular}{|c|c|c|c|}
\hline \multirow[t]{2}{*}{ Outcomes } & \multicolumn{2}{|c|}{ Period [median (IQR) or $n(\%)]$} & \multirow[t]{2}{*}{$P$ value } \\
\hline & During Ramadan & After Ramadan & \\
\hline \multicolumn{4}{|l|}{ Questionnaire (h) } \\
\hline \multicolumn{4}{|l|}{ Daily total sleeping hours } \\
\hline$<6$ & $4(11.1 \%)$ & $2(5.6 \%)$ & \multirow[t]{3}{*}{0.007} \\
\hline $6-8$ & $27(75 \%)$ & $18(50 \%)$ & \\
\hline$>8$ & $5(13.9 \%)$ & $16(44.4 \%)$ & \\
\hline \multicolumn{4}{|l|}{ Daily night-time sleeping hours } \\
\hline Never & $20(55.6 \%)$ & $3(8.3 \%)$ & \multirow[t]{4}{*}{0.005} \\
\hline$>3$ & $5(13.9 \%)$ & $9(25 \%)$ & \\
\hline $3-6$ & $6(16.7 \%)$ & $14(38.9 \%)$ & \\
\hline$>6$ & $5(13.9 \%)$ & $10(27.8 \%)$ & \\
\hline \multicolumn{4}{|l|}{ Fitbit Flex $2(\mathrm{~h})$} \\
\hline Average daily total sleeping hours $(\mathrm{h})$ & $6.03(5.37-6.67)$ & $7.02(6.35-7.71)$ & $<0.001$ \\
\hline \multicolumn{4}{|l|}{ Average daily total sleeping hours categories } \\
\hline$<6$ & $18(50 \%)$ & $5(13.9 \%)$ & \multirow[t]{3}{*}{$<0.001$} \\
\hline $6-8$ & $17(47.2 \%)$ & $25(69.4 \%)$ & \\
\hline$>8$ & $1(2.8 \%)$ & $6(16.7 \%)$ & \\
\hline Average daily night-time sleeping hours $(\mathrm{h})$ & $0(0-1.30)$ & $1.78(0.67-2.88)$ & $<0.001$ \\
\hline \multicolumn{4}{|c|}{ Average daily night-time sleeping hours categories } \\
\hline Never & $18(50 \%)$ & $6(16.7 \%)$ & \multirow[t]{4}{*}{$<0.001$} \\
\hline$>3$ & $17(47.2 \%)$ & $22(61.1 \%)$ & \\
\hline $3-6$ & $1(2.8 \%)$ & $7(19.4 \%)$ & \\
\hline$>6$ & $0(0 \%)$ & $1(2.8 \%)$ & \\
\hline
\end{tabular}

$P$ values for non-parametric paired Wilcoxon signed-rank tests $S D$ standard deviation, $n$ number

For example, the Saudi General Authority for Statistics reported that $82.6 \%$ of Saudis did not practice physical activity for $150 \mathrm{~min}$ or more per week in 2018 [12]. Furthermore, the World Health Organization (WHO) reported in 2016 that the prevalence of physical inactivity in the Saudi population was $58.5 \%$ [49].

The high prevalence of physical inactivity and high sedentary time observed in the current study could be attributed to some barriers. For instance, the climate has been previously found 
by Amin et al. [50] to be an obstacle for $65.9 \%$ of Saudis to perform physical activity. In addition, climate prevented outdoor activity in $80 \%$ of Saudi individuals with T2DM in Riyadh [51]. The climate prevents people from performing physical activity, especially outdoor activities, because of the hot temperature outside. However, the establishment of community sports centres with adapted indoor temperature can minimise the negative impact of climate on physical activity. Unfortunately, the lack of these centres has been reported as a barrier to performing physical activity among Saudis $[52,53]$, which could be another possible explanation for the finding of this study for the low level of physical activity. Furthermore, the lack of time in Saudis has been reported by previous researchers as a barrier for performing physical activity [50, 52]. Another potential impediment could be urbanisation [52, 54], whereby Saudis living in villages were found to be more physically active than who live in cities, which can be attributed to the participation in some farming tasks such as ploughing and planting [55].

Another interesting finding of the current study is that no statistically significant differences in physical activity were detected between the during and after Ramadan periods, which agrees with previous studies that did not report changes in physical activity of individuals with T2DM during Ramadan [31, 56]. In healthy individuals from Riyadh, no differences were observed in their physical activity during Ramadan compared to before or after Ramadan [57]. Although the current study did not detect significant differences in the participants' activities between periods, physical activity during Ramadan was slightly higher than in the after Ramadan period. A similar finding has been reported by Sfar et al. [58], who showed a non-significant increase in physical activity of Tunisian individuals with T2DM during Ramadan compared to the pre-Ramadan period. In addition, the total activity measured by IPAQ showed a significant increase during Ramadan in this study compared to the after Ramadan period, but with small effect size. This non-significant increase of physical activity during Ramadan could be attributed to the increase in prayer activities such as Taraweeh prayers, additional ritual prayers performed by Muslims at night during Ramadan, which was previously suggested as a form of "physical activity" due to the long hours of standing during prayers [59]. However, there is the possibility that the "after" Ramadan results are not much different because a 2-week period may not be enough to change behaviours.

The current study showed strong positive correlations between the measurements of physical activity by IPAQ and Fitbit Flex 2 accelerometer. In addition, previous studies have reported that Fitbit devices are quite reliable for assessing physical activity $[35,36,60,61]$. Hence, this finding of the current study reveals that the IPAQ was acceptable and reliable for measuring the physical activity of its participants. However, this study also indicated that the sitting time measured by IPAQ was under-reported by participants compared to the sedentary time measured by the Fitbit device. This could be due to misreporting, because a previous study concluded that the sitting time determined by IPAQ was under-reported by the study population when compared to the sedentary time recorded by an accelerometer [62]. In addition, self-reported surveys are susceptible to underestimating or misreporting of the sedentary time by the participants as a result of recall bias [63, 64].

The statistical analysis of the current study detected significant differences between the during and after Ramadan periods in the sleeping hours obtained by both instruments. These differences in the current study were observed with significantly shorter sleeping hours during Ramadan compared to the after Ramadan period. Although $7-8 \mathrm{~h}$ of daily sleeping is the recommended duration for optimal health $[13,65,66]$, the daily average of sleeping among half the participants during Ramadan was less than $6 \mathrm{~h}$. This finding agrees with the previous studies that reported a reduction in sleep durations during Ramadan [22, 24, 67, 68]. Furthermore, insufficient sleep duration of less than $8 \mathrm{~h}$ has been previously observed among $95 \%$ of the participants of a study conducted on Saudi female students from a college in Riyadh [69]. The amount of sleeping at night-time, 
reported by the latter study, was found to be low in both periods. The duration of night-time sleep significantly rose after Ramadan compared to the during Ramadan period. Previous studies have demonstrated similar findings of short amounts of night-time sleep during Ramadan [25-27], and short night-time sleep duration and excessive daytime tiredness among the Saudi population is not only limited to the month of Ramadan but is seen throughout the year $[16,17]$.

The changes in meals times, because Muslims abstain from eating and drinking during the daytime of Ramadan, could be the possible reason for the shortened sleep duration during Ramadan especially at night-time [22]. In addition, other environmental factors, such as higher daytime temperature, and stores and shopping malls remaining open until late during the Ramadan period could be considered as other possible explanations for the shortened night-time sleep during Ramadan. The practice of additional worships including Taraweeh prayers during the night-time of Ramadan could be another possible reason for this finding. Hence, the shortened night-time sleep during Ramadan could be the reason behind the shortened daily total sleeping hours during Ramadan compared to the after Ramadan period. The after Ramadan period of the current study occurred during the summer holiday, in which the longer night-time is usually spent with family and friends because of the events and parties, which is a likely explanation for the short night-time sleep hours in this period.

Although the self-reported method for sleep quality has been previously found to be positively correlated with the data obtained by Fitbit [61], the current study did not detect significant correlations between both methods in each period. The participants of the current study overestimated their sleeping hours in the questionnaires compared to the results recorded by the Fitbit devices. This contradictory finding is likely because the measurement of sleep is prone to recall bias and misreporting by participants in self-reported measures [64]. However, it is important to note that these explanations do not absolutely mean that Fitbit measurements are accurate; they have been previously reported to have insufficient accuracy in assessing sleep patterns compared to polysomnography [70].

This study demonstrated that the means of HbA1c levels were more than $7 \%$ in each period, which is considered as poor glycaemic control according to the guidelines of the American Diabetes Association [71]. The observed behaviours of physical inactivity and poor sleeping pattern could be one of the possible reasons behind this poor control. Additionally, the mean HbA1c levels were lower in the during Ramadan compared to after Ramadan period although the change is neither statistically significant nor clinically important. Previous studies have reported that intermittent fasting is beneficial for individuals with T2DM [41-43], and reversed T2DM in some cases [44, 45]. The month of Ramadan involves a type of religious intermittent fasting, when Muslims abstain from eating, drinking, smoking, and sexual interaction from dawn to dusk for 29 or 30 days [21], which also has been previously reported to have a positive impact on T2DM [28-31]. However, the month of Ramadan is associated with significant changes in lifestyle, such as altered sleeping durations and times and changes in physical activities [22, 24]. Indeed, physical activity and sleeping quality have been demonstrated by numerous studies as important factors that influence the outcomes of T2DM [2-6]. Increased physical activity has been indicated to be associated with reduced HbA1c levels [9]. In addition, the disturbance of the circadian rhythm has been previously reported to be a risk factor for developing T2DM by causing insulin resistance $[19,20]$. Makino et al. [72] reported that night-time sleeping hours of less than $5 \mathrm{~h}$ per night were positively correlated with an increase in HbA1c levels in Japanese individuals with T2DM when compared to longer night-time sleeping hours. In line with these findings, it can be concluded that decrease in sleeping hours during Ramadan, observed in our study, could minimize the positive impact of fasting on people with T2DM. However, it is possible that the 2-week period after the end of Ramadan, when HbA1c was measured, might not be enough to detect significant differences. 


\section{STRENGTHS}

To the best of our knowledge, this study is the first to investigate and evaluate the physical activity and sleeping patterns of individuals with type 2 diabetes fasting in the during and after Ramadan periods, using subjective and objective techniques (IPAQ and Fitbit Flex 2). The application of wearable technology to assess changes in lifestyle during Ramadan fasting can help individuals and health professionals to efficiently track or monitor physical activity and sleep patterns, and to generate accurate and reliable results for the management of disease and improve public health.

\section{LIMITATIONS}

Unfortunately, this study is subject to some limitations. Since the current study was an exploratory pilot study with a relatively small sample size, it has limited statistical power. However, the obtained results are highly informative and call for further cohort studies to verify the importance of the findings of the current study. In addition, it is not possible to confirm that every participant used the Fitbit device properly for the entire study duration, or each device recorded or synced all activities, which could affect the overall results. Moreover, as with most self-reported measurements, the questionnaires used in this study were also subject to bias, which affected the reliability of the results. For example, the participants reported that they fasted during Ramadan but there is no way of verifying if this is true as fasting is a very personal matter. Furthermore, the used questionnaires for the assessment of sleep patterns may not have been sufficient. Additionally, although the participants recruited were Saudis from Riyadh, the small sample size means that they are not representative of the entire Saudi population diagnosed with T2DM. For this to be the case, more participants from different regions of Saudi Arabia would have been required for further cohort studies. Another important limitation was the lack of a control group, as this would add strong evidence to the overall conclusion. In addition, a 2-week period after the end of Ramadan may not be sufficient to detect significant changes. The before Ramadan period was unfortunately missed since the ethical approval was obtained later. Therefore, conducting a larger cohort study that includes measurements before, during, and after Ramadan could provide a better picture about how these behaviours change (or not) as a result of fasting.

\section{CONCLUSIONS}

This is the first study to combine a wearable device (Fitbit) and a self-reported questionnaire to monitor physical activity and sleeping patterns in individuals with T2D who chose to fast during the month of Ramadan. We can conclude that a high prevalence of physical inactivity was noticed among individuals with T2DM in the during and after Ramadan periods, with no significant differences between these periods regarding their physical activity. This study also indicates that the daily total sleeping hours significantly varied between the during and after Ramadan periods, with significantly shorter sleep durations observed in the during Ramadan fasting period. Although the prevalence of short night-time sleeping hours was nevertheless high for most individuals in both periods, the during Ramadan period showed significant short night-time sleeping hours. In addition, this study reveals that the data recorded by the Fitbit Flex 2 accelerometer were significantly correlated with the self-reported outcomes obtained by IPAQ for the physical activity of individuals, but not for sleeping patterns collected by a self-reported questionnaire. Therefore, the low physical activity and the poor sleep behaviours of the study's participants may worsen their diabetes, and minimise the potential of Ramadan intermittent fasting as a non-pharmacological therapy for management of T2DM. A larger study is needed in the future covering before, during, and after Ramadan to fully assess the impact of Ramadan on T2DM. 


\section{ACKNOWLEDGEMENTS}

The authors would like to thank the General Directorate of Medical Services of the Interior Ministry in Saudi Arabia for supporting Abdullah Alghamdi for a PhD Scholarship. Authors acknowledge all doctors, nurses, staffs, and all members of the Endocrine and Diabetes Centre, Al-Iman General Hospital, Riyadh for their help during the conduct of this research. The authors would like also to thank the main laboratory of Al Iman General Hospital for measuring the blood samples. Authors thank all the participants who took part in this study.

Funding. The journals Rapid Service Fee for the publication of this study was provided by De Montfort University from the scholarship fund of Abdullah Alghamdi.

Authorship. All named authors meet the International Committee of Medical Journal Editors (ICMJE) criteria for authorship for this article, take responsibility for the integrity of the work as a whole, and have given their approval for this version to be published..

Disclosures. Abdullah S. Alghamdi, Khalid A. Alghamdi, Richard O. Jenkins, Mohammed N. Alghamdi and Parvez I. Haris have nothing to disclose.

Compliance with Ethics Guidelines. Ethical approvals were obtained from the Faculty of Health and Life Sciences Research Ethics Committee at De Montfort University and the Ministry of Health in Saudi Arabia. All procedures followed were in accordance with the Helsinki Declaration of 1964 and its later amendments. Informed consent was obtained from each participant prior to commencement of the study to take part in this study and for any publication with non-identifiable quotes.

Data Availability. The datasets generated during and/or analysed during the current study are available from the corresponding author on reasonable request.
Open Access. This article is licensed under a Creative Commons Attribution-NonCommercial 4.0 International License, which permits any non-commercial use, sharing, adaptation, distribution and reproduction in any medium or format, as long as you give appropriate credit to the original author(s) and the source, provide a link to the Creative Commons licence, and indicate if changes were made. The images or other third party material in this article are included in the article's Creative Commons licence, unless indicated otherwise in a credit line to the material. If material is not included in the article's Creative Commons licence and your intended use is not permitted by statutory regulation or exceeds the permitted use, you will need to obtain permission directly from the copyright holder. To view a copy of this licence, visit http://creativecommons.org/licenses/by$\mathrm{nc} / 4.0 /$.

\section{REFERENCES}

1. Meo SA, Usmani AM, Qalbani E. Prevalence of type 2 diabetes in the Arab world: impact of GDP and energy consumption. Eur Rev Med Pharmacol Sci. 2017;21(6):1303-12.

2. Heath GW, Gavin JR 3rd, Hinderliter JM, Hagberg JM, Bloomfield SA, Holloszy JO. Effects of exercise and lack of exercise on glucose tolerance and insulin sensitivity. J Appl Physiol Respir Environ Exerc Physiol. 1983;55(2):512-7.

3. Lee IM, Shiroma EJ, Lobelo F, et al. Effect of physical inactivity on major non-communicable diseases worldwide: an analysis of burden of disease and life expectancy. Lancet. 2012;380(9838):219-29.

4. Tsai YW, Kann NH, Tung TH, et al. Impact of subjective sleep quality on glycemic control in type 2 diabetes mellitus. Fam Pract. 2012;29(1):30-5.

5. Gottlieb DJ, Punjabi NM, Newman AB, et al. Association of sleep time with diabetes mellitus and impaired glucose tolerance. Arch Intern Med. 2005;165(8):863-7.

6. Zimmet P, Alberti KG, Shaw J. Global and societal implications of the diabetes epidemic. Nature. 2001;414(6865):782-7.

7. Colberg SR, Sigal RJ, Fernhall B, et al. Exercise and type 2 diabetes: the American College of Sports 
Medicine and the American Diabetes Association: joint position statement. Diabetes Care. 2010;33(12):e147-e167167.

8. Look ARG, Wing RR. Long-term effects of a lifestyle intervention on weight and cardiovascular risk factors in individuals with type 2 diabetes mellitus: four-year results of the look AHEAD trial. Arch Intern Med. 2010;170(17):1566-75.

9. Kooiman TJM, de Groot M, Hoogenberg K, Krijnen WP, van der Schans CP, Kooy A. Self-tracking of physical activity in people with type 2 diabetes: a randomized controlled trial. Comput Inform Nurs. 2018;36(7):340-9.

10. Ades PA, Savage PD, Toth MJ, et al. High-calorieexpenditure exercise: a new approach to cardiac rehabilitation for overweight coronary patients. Circulation. 2009;119(20):2671-8.

11. Cowie CC, Harris MI, Silverman RE, Johnson EW, Rust KF. Effect of multiple risk factors on differences between blacks and whites in the prevalence of non-insulin-dependent diabetes mellitus in the United States. Am J Epidemiol. 1993;137(7):719-32.

12. General Authority for Statistics. Bulletin of Household Sport Practice Survey. Kingdom of Saudi Arabia. 2018. https://www.stats.gov.sa/sites/default/ files/household_sport_practice_survey_2018_en. pdf.

13. Lee SWH, Ng KY, Chin WK. The impact of sleep amount and sleep quality on glycemic control in type 2 diabetes: a systematic review and metaanalysis. Sleep Med Rev. 2017;31:91-101.

14. Brocato J, Wu F, Chen Y, et al. Association between sleeping hours and cardiometabolic risk factors for metabolic syndrome in a Saudi Arabian population. BMJ Open. 2015;5(11):e008590.

15. Jauch-Chara K, Hallschmid M, Schmid SM, et al. Plasma glucagon decreases during night-time sleep in type 1 diabetic patients and healthy control subjects. Diabet Med. 2007;24(6):684-7.

16. Fatani A, Al-Rouqi K, Al Towairky J, et al. Effect of age and gender in the prevalence of excessive daytime sleepiness among a sample of the Saudi population. J Epidemiol Glob Health. 2015;5(4 Suppl 1):S59-66.

17. Ahmed AE, Al-Jahdali F, Al AA, et al. Prevalence of sleep duration among Saudi adults. Saudi Med J. 2017;38(3):276-83.

18. Bahijri S, Borai A, Ajabnoor G, et al. Relative metabolic stability, but disrupted circadian cortisol secretion during the fasting month of Ramadan. PLoS One. 2013;8(4):e60917.
19. Ajabnoor GMA, Bahijri S, Shaik NA, et al. Ramadan fasting in Saudi Arabia is associated with altered expression of CLOCK, DUSP and IL-1alpha genes, as well as changes in cardiometabolic risk factors. PLoS One. 2017;12:e0174342.

20. Ajabnoor GM, Bahijri S, Borai A, Abdulkhaliq AA, Al-Aama JY, Chrousos GP. Health impact of fasting in Saudi Arabia during Ramadan: association with disturbed circadian rhythm and metabolic and sleeping patterns. PLoS One. 2014;9(5):e96500.

21. The Holy Quran. Surah 2 (Al-Baqarah), Verse 185.

22. Bahammam A. Does Ramadan fasting affect sleep? Int J Clin Pract. 2006;60(12):1631-7.

23. Sadiya A, Ahmed S, Siddieg HH, Babas IJ, Carlsson M. Effect of Ramadan fasting on metabolic markers, body composition, and dietary intake in Emiratis of Ajman (UAE) with metabolic syndrome. Diabetes Metab Syndr Obes. 2011;4:409-16.

24. Lessan N, Saadane I, Alkaf B, et al. The effects of Ramadan fasting on activity and energy expenditure. Am J Clin Nutr. 2018;107(1):54-61.

25. Bahammam A. Effect of fasting during Ramadan on sleep architecture, daytime sleepiness and sleep pattern. Sleep Biol Rhythms. 2004;2(2):135-43.

26. BaHammam A. Assessment of sleep patterns, daytime sleepiness, and chronotype during Ramadan in fasting and nonfasting individuals. Saudi Med J. 2005;26(4):616-22.

27. Taoudi MB, Roky R, Toufiq J, Benaji B, Hakkou F. Epidemiological study: chronotype and daytime sleepiness before and during Ramadan. Therapie. 1999;54(5):567-72.

28. Sahin SB, Ayaz T, Ozyurt N, Ilkkilic K, Kirvar A, Sezgin $\mathrm{H}$. The impact of fasting during Ramadan on the glycemic control of patients with type 2 diabetes mellitus. Exp Clin Endocrinol Diabetes. 2013;121(9):531-4.

29. Karatoprak C, Yolbas S, Cakirca M, et al. The effects of long term fasting in Ramadan on glucose regulation in type 2 diabetes mellitus. Eur Rev Med Pharmacol Sci. 2013;17(18):2512-6.

30. Bener A, Al-Hamaq AO, Ozturk M, et al. Effect of ramadan fasting on glycemic control and other essential variables in diabetic patients. Ann Afr Med. 2018;17(4):196-202.

31. Khatib FA, Shafagoj YA. Metabolic alterations as a result of Ramadan fasting in non-insulin-dependent diabetes mellitus patients in relation to food intake. Saudi Med J. 2004;25(12):1858-63. 
32. Shephard RJ. Limits to the measurement of habitual physical activity by questionnaires. Br J Sports Med. 2003;37(3):197-206.

33. Motl RW, McAuley E, DiStefano C. Is social desirability associated with self-reported physical activity? Prev Med. 2005;40(6):735-9.

34. Althubaiti A. Information bias in health research: definition, pitfalls, and adjustment methods. J Multidiscip Healthc. 2016;9:211-7.

35. Ferguson T, Rowlands AV, Olds T, Maher C. The validity of consumer-level, activity monitors in healthy adults worn in free-living conditions: a cross-sectional study. Int J Behav Nutr Phys Act. 2015;12:42.

36. Vooijs M, Alpay LL, Snoeck-Stroband JB, et al. Validity and usability of low-cost accelerometers for internet-based self-monitoring of physical activity in patients with chronic obstructive pulmonary disease. Interact J Med Res. 2014;3(4):e14.

37. Fitbit.com. Fitbit Flex $2^{\mathrm{TM}}$ Fitness Wristband: Fitbit.com. https://www.fitbit.com/uk/flex2 (2019). Accessed 03 Nov 2019

38. International Physical Activity Questionnaires (IPAQ). https://www.ipaq.ki.se (2018). Accessed 28 Nov 2018.

39. Craig CL, Marshall AL, Sjostrom M, et al. International physical activity questionnaire: 12-country reliability and validity. Med Sci Sports Exerc. 2003;35(8):1381-95.

40. Cohen J. A power primer. Psychol Bull. 1992;112(1):155.

41. Corley BT, Carroll RW, Hall RM, Weatherall M, Parry-Strong A, Krebs JD. Intermittent fasting in type 2 diabetes mellitus and the risk of hypoglycaemia: a randomized controlled trial. Diabet Med. 2018;35(5):588-94.

42. Cheng CW, Villani V, Buono R, et al. Fastingmimicking diet promotes Ngn3-driven beta-cell regeneration to reverse diabetes. Cell. 2017;168(5): 775-888.

43. Diabetes Prevention Program Research Group. The Diabetes Prevention Program (DPP): description of lifestyle intervention. Diabetes Care. 2002;25(12): 2165-71.

44. $\mathrm{Ku} \mathrm{M}$, Ramos MJ, Fung J. Therapeutic fasting as a potential effective treatment for type 2 diabetes : a 4-month case study. J Insul Resist. 2017;2:a31. https://doi.org/10.4102/jir.v2i1.31
45. Carter S, Clifton PM, Keogh JB. Effect of intermittent compared with continuous energy restricted diet on glycemic control in patients with type 2 diabetes: a randomized noninferiority trial. JAMA Netw Open. 2018;1(3):e180756.

46. Yates T, Khunti K, Wilmot EG, et al. Self-reported sitting time and markers of inflammation, insulin resistance, and adiposity. Am J Prev Med. 2012;42(1):1-7.

47. Healy GN, Winkler EA, Brakenridge CL, Reeves MM, Eakin EG. Accelerometer-derived sedentary and physical activity time in overweight/obese adults with type 2 diabetes: cross-sectional associations with cardiometabolic biomarkers. PLoS One. 2015;10(3):e0119140.

48. Richter EA, Ploug T, Galbo H. Increased muscle glucose uptake after exercise No need for insulin during exercise. Diabetes. 1985;34(10):1041-8.

49. World Health Organization. Diabetes country profiles, 2016-Saudi Arabia. 2016. https://www.who. int/diabetes/country-profiles/sau_en.pdf?ua=1.

50. Amin TT, Suleman W, Ali A, Gamal A, Al WA. Pattern, prevalence, and perceived personal barriers toward physical activity among adult Saudis in AlHassa, KSA. J Phys Act Health. 2011;8:775-84.

51. Mogahed M. Vitamin D status in patients with type2 diabetes mellitus in Riyadh City, Saudi Arabia. Kasr Al Ainy Med J. 2018;24(1):19-25.

52. Al-Rafaee SA, Al-Hazzaa HM. Physical activity profile of adult males in Riyadh City. Saudi Med J. 2001;22(9):784-9.

53. Alsubaie ASR, Omer EOM. Physical activity behavior predictors, reasons and barriers among male adolescents in Riyadh, Saudi Arabia: evidence for obesogenic environment. Int $\mathrm{J}$ Health Sci. 2015;9(4):395-402.

54. Al-Hazzaa HM. Physical inactivity in Saudi Arabia revisited: a systematic review of inactivity prevalence and perceived barriers to active living. Int $\mathrm{J}$ Health Sci. 2018;12(6):50-64.

55. Al-Nuaim AA, Al-Nakeeb Y, Lyons $\mathrm{M}$, et al. The prevalence of physical activity and sedentary behaviours relative to obesity among adolescents from Al-Ahsa, Saudi Arabia: rural versus urban variations. J Nutr Metab. 2012;2012:417589.

56. Siaw MY, Chew DE, Dalan R, et al. Evaluating the effect of ramadan fasting on muslim patients with diabetes in relation to use of medication and lifestyle patterns: a prospective study. Int J Endocrinol. 2014;2014:308546. 
57. Al-Barha NS, Aljaloud KS. The effect of Ramadan fasting on body composition and metabolic syndrome in apparently healthy men. Am J Mens Health. 2019;13(1):1557988318816925.

58. Sfar H, Sellami S, Boukhayatia F, Naceur K, Mami F. Biochemical, physiological and body composition changes in patients with type 2 diabetes during Ramadan fasting. Ibnosina J Med Biomed Sci. 2017;9(6):164-8.

59. Ibrahim M, Al Magd MA, Annabi FA, et al. Recommendations for management of diabetes during Ramadan: update 2015. BMJ Open Diabetes Res Care. 2015;3(1):e000108.

60. Brewer W, Swanson BT, Ortiz A. Validity of Fitbit's active minutes as compared with a research-grade accelerometer and self-reported measures. BMJ Open Sport Exerc Med. 2017;3(1):e000254.

61. Evenson KR, Goto MM, Furberg RD. Systematic review of the validity and reliability of consumerwearable activity trackers. Int J Behav Nutr Phys Act. 2015;12:159.

62. Dyrstad SM, Hansen BH, Holme IM, Anderssen SA. Comparison of self-reported versus accelerometermeasured physical activity. Med Sci Sports Exerc. 2014;46(1):99-106.

63. Klesges RC, Eck LH, Mellon MW, Fulliton W, Somes GW, Hanson CL. The accuracy of self-reports of physical activity. Med Sci Sports Exerc. 1990;22(5): 690-7.

64. Ainsworth B, Cahalin L, Buman M, Ross R. The current state of physical activity assessment tools. Prog Cardiovasc Dis. 2015;57(4):387-95.
65. Ferrie JE, Shipley MJ, Cappuccio FP, et al. A prospective study of change in sleep duration: associations with mortality in the Whitehall II cohort. Sleep. 2007;30(12):1659-66.

66. Wickboldt AT, Bowen AF, Kaye AJ, Kaye AM, Rivera Bueno F, Kaye AD. Sleep physiology, abnormal States, and therapeutic interventions. Ochsner J. 2012;12(2):122-34.

67. Herrera CP. Total sleep time in Muslim football players is reduced during Ramadan: a pilot study on the standardized assessment of subjective sleepwake patterns in athletes. J Sports Sci. 2012;30(Suppl 1):S85-91.

68. Roky R, Chapotot F, Hakkou F, Benchekroun MT, Buguet A. Sleep during Ramadan intermittent fasting. J Sleep Res. 2001;10(4):319-27.

69. Alzamil HA, Alhakbany MA, Alfadda NA, Almusallam SM, Al-Hazzaa HM. A profile of physical activity, sedentary behaviors, sleep, and dietary habits of saudi college female students. J Fam Community Med. 2019;26(1):1-8.

70. Moreno-Pino F, Porras-Segovia A, Lopez-Esteban P, Artes A, Baca-Garcia E. Validation of Fitbit charge 2 and Fitbit Alta HR against polysomnography for assessing sleep in adults with obstructive sleep apnea. J Clin Sleep Med. 2019;15(11):1645-53.

71. American Diabetes Association. 6. Glycemic targets: standards of medical care in diabetes-2019. Diabetes Care. 2019;42(Suppl 1):S61-S70.

72. Makino S, Hirose S, Kakutani M, et al. Association between nighttime sleep duration, midday naps, and glycemic levels in Japanese patients with type 2 diabetes. Sleep Med. 2018;44:4-11. 